This article was downloaded by:[Swets Content Distribution]

On: 27 July 2007

Access Details: [subscription number 768307933]

Publisher: Psychology Press

Informa Ltd Registered in England and Wales Registered Number: 1072954

Registered office: Mortimer House, 37-41 Mortimer Street, London W1T 3JH, UK

\title{
APHASIOLOGY Aphasiology
}

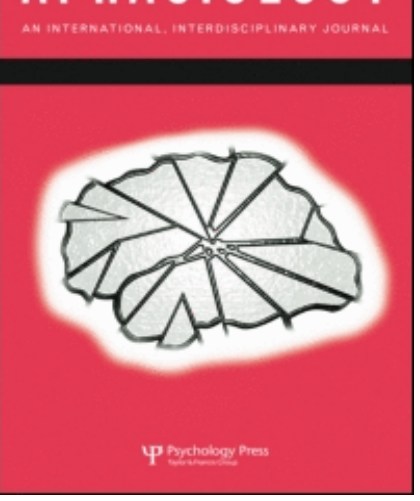

Publication details, including instructions for authors and subscription information: http://www.informaworld.com/smpp/title content=t713393920

Progressive non-fluent aphasia is not a progressive form of non-fluent (post-stroke) aphasia

Karalyn Patterson a; Naida L. Graham a; Matthew A. Lambon Ralph b; John R. Hodges ${ }^{\text {a }}$

a MRC Cognition \& Brain Sciences Unit, Cambridge, UK

b University of Manchester, UK

Online Publication Date: 01 September 2006

To cite this Article: Patterson, Karalyn, Graham, Naida L., Ralph, Matthew A. Lambon and Hodges, John R. (2006) 'Progressive non-fluent aphasia is not a progressive form of non-fluent (post-stroke) aphasia ', Aphasiology, 20:9, 1018 1034

To link to this article: DOI: $10.1080 / 02687030600739463$

URL: http://dx.doi.org/10.1080/02687030600739463

\section{PLEASE SCROLL DOWN FOR ARTICLE}

Full terms and conditions of use: http://www.informaworld.com/terms-and-conditions-of-access.pdf

This article maybe used for research, teaching and private study purposes. Any substantial or systematic reproduction, re-distribution, re-selling, loan or sub-licensing, systematic supply or distribution in any form to anyone is expressly forbidden.

The publisher does not give any warranty express or implied or make any representation that the contents will be complete or accurate or up to date. The accuracy of any instructions, formulae and drug doses should be independently verified with primary sources. The publisher shall not be liable for any loss, actions, claims, proceedings, demand or costs or damages whatsoever or howsoever caused arising directly or indirectly in connection with or arising out of the use of this material.

(C) Taylor and Francis 2007 


\title{
Progressive non-fluent aphasia is not a progressive form of non-fluent (post-stroke) aphasia
}

\author{
Karalyn Patterson and Naida L. Graham \\ MRC Cognition \& Brain Sciences Unit, Cambridge, UK \\ Matthew A. Lambon Ralph \\ University of Manchester, UK \\ John R. Hodges \\ MRC Cognition \& Brain Sciences Unit, Cambridge, UK
}

Background: The speech of patients with progressive non-fluent aphasia (PNFA) has been described as similar to that in non-fluent aphasia (NFA) consequent on stroke. There are, however, few direct empirical comparisons of these two patient populations in the literature.

Aims: To test the hypotheses that PNFA cases differ from NFA (a) in the extent to which their speech production deficit varies as a function of speaking task, and (b) in the nature of their phonological deficit.

Methods \& Procedures: Groups of PNFA and NFA patients $(N=10$ each), matched on scores in a picture-naming test, were assessed on tasks of narrative picture description, reading aloud of text and single words, and phonological abilities such as rhyme judgement and rhyme production.

Outcomes \& Results: (a) The NFA cases showed equivalent speech rates in selfgenerated speech and reading text aloud, and equivalent error rates when reading text or isolated single words. In contrast, the PNFA cases spoke more rapidly when reading aloud than when producing narrative speech, and achieved higher accuracy when reading single words aloud than when reading words in text. (b) Variation in success rate for reading different types of words (e.g., content words, function words, and nonsense words), error types in reading, and performance on phonological tasks all indicated a different and better quality of phonological processing in PNFA than NFA.

Address correspondence to: Karalyn Patterson, MRC Cognition and Brain Sciences Unit, 15 Chaucer Road, Cambridge, CB2 2EF, UK. E-mail: karalyn.patterson@mrc-cbu.cam.ac.uk

This contribution, like the others in this Special Issue, is dedicated to John C. Marshall: he and his work have been a considerable inspiration to all of the authors of this paper.

This research was supported in part by a (USA) NIMH Interdisciplinary Behavioural Science Centre Grant (No. MH64445: KP, NLG and MALR) and by a (UK) MRC Programme Grant (No. G9724461: JRH).

We are grateful to Natalie Braber and Katherine Ellis for assistance with data collection for the NFA cases, to Peter Watson for statistical assistance, and to Peter Nestor for helpful discussions regarding the PNFA patients. 
Conclusions: Despite some surface similarities, there are telling differences between the speech impairments in PNFA and NFA. The deficit in PNFA particularly compromises self-generated connected speech.

Descriptions of non-fluent aphasia resulting from left-hemisphere cerebrovascular accident (CVA) have been in the literature since the time of Broca (1861). Some 120 years later, a similar form of language disorder resulting from neurodegenerative disease was brought to widespread attention by Mesulam (1982, 2001). Although some authors continue to use Mesulam's terminology of "primary progressive aphasia" without specifying speech character, others have now adopted the label progressive non-fluent aphasia to refer to the pattern of slowly progressive aphasia in which speech output is notably dysfluent (Gorno-Tempini et al., 2004; Grossman \& Ash, 2005; Hodges \& Patterson, 1996; Snowden, Neary, \& Mann, 1996; Thompson, Ballard, Tait, Weintraub, \& Mesulam, 1997). Thus, there are two syndrome labels, non-fluent aphasia (NFA) and progressive non-fluent aphasia (PNFA), differing only in the presence/absence of the adjective progressive. These labels give the impression that - apart from the fact that one of these disorders emerges abruptly after a CVA whereas the other develops gradually as a consequence of a neurodegenerative condition - they are two versions of a single aphasic profile.

Furthermore, it is not only the labels that foster this view: although almost all discussions of PNFA comment on the heterogeneity of the language disorder, the pattern most commonly described is effortful, dysfluent speech coupled with phonological and syntactic impairments, which is the typical picture of NFA (Brown, 1972; Caplan, 1987; Saffran, Schwartz, \& Marin, 1980). For example, Gorno-Tempini et al. (2004, p. 335) characterised PNFA as comprising “... laboured speech, agrammatism in production and/or comprehension, variable degrees of anomia, and phonemic paraphasias, in the presence of relatively preserved word comprehension". Unlike several large-scale comparisons of PNFA with language disruption in Alzheimer's disease (Grossman et al., 1996; Kertesz, Davidson, McCabe, Takagi, \& Munoz, 2003; Mendez, Clark, Shapira, \& Cummings, 2003), the literature offers few direct and empirical (as opposed to descriptive) comparisons between PNFA and NFA from stroke. In one major exception (Thompson et al., 1997), the authors used a variety of linguistic measures in the realms of both word and sentence processing, and concluded that three of the four cases of PNFA that they tracked longitudinally had a pattern resembling the NFA cases in their study.

Our own observations have led to the hypotheses that there are at least three potentially salient differences between the language disorders in these two conditions. One of these pertains to the claim that the expressive language of patients with PNFA is typically agrammatic. Contrary to this view, Graham, Patterson, and Hodges (2004) have recently reported that the narrative speech (as measured by picture description) of a group of 14 PNFA cases, relative to that of age-matched controls, was reduced in quantity but had normal ratios of verbs to nouns and of function words to content words. The remaining two hypothesised differences are explored in the current study comparing PNFA with NFA. First, a set of tasks was designed to address the hypothesis that the speech production deficit in PNFA, whilst not exactly milder than that in NFA, is significantly more modifiable by the nature of the language task. More specifically, we predicted that the deficit in PNFA, by contrast to NFA, would primarily disrupt performance in 
tasks requiring self-generated connected language. Second, we predicted marked differences in the nature of the phonological deficit in PNFA versus NFA.

\section{METHOD}

\section{Statistical analyses}

The majority of statistical tests reported in this paper, apart from a few correlations, are non-parametric two-tailed Mann-Whitney $U$ tests (for between-group contrasts) and Wilcoxon tests (for within-group comparisons) because of inhomogeneity of variance in the clinical populations.

\section{Participants}

Two groups of patients were the source of the data reported here. The first comprised 10 right-handed individuals who had suffered a single left-hemisphere CVA, referred by several consultant neurologists and speech therapists in both Cambridge and Manchester, UK. They were selected for this study on the basis of slow effortful speech containing phonological and/or phonetic errors. Five of these cases had participated in a set of experiments on past-tense verb inflection in nonfluent aphasia (Bird, Lambon Ralph, Seidenberg, McClelland, \& Patterson, 2003).

The second experimental group comprised 10 PNFA patients (9 right-handed, 1 left-handed) selected from a slightly larger sample $(N=14$, reported in Graham et al., 2004) with the goal of matching to the 10 NFA cases just described. They were all recruited from either the Memory Disorders Clinic or the Early Dementia Clinic at Addenbrooke's Hospital, Cambridge, UK, where they presented with a predominant complaint of speech difficulty. In varying degrees, all had slowed, effortful output with phonological errors in spontaneous speech or on formal testing. Every patient in this sub-group has deteriorated on follow-up, supporting the progressive diagnosis. MRI in all cases ruled out stroke, and was often rather unremarkable apart from a degree of abnormal widening of the left Sylvian fissure. Of the 10 reported here, 5 were included in a study of resting cerebral metabolism (FDG-PET), which demonstrated that the most significant region of hypometabolism was the left anterior insula/frontal operculum (Nestor, Graham, Fryer, Williams, Patterson, \& Hodges, 2003). In general, the patients in this group fulfilled the standard Mesulam criterion of at least a 2-year history of language disruption in the context of preserved activities of daily living. None of the cases had a clear dementia, although neuropsychological testing revealed a range of function.

It was not obvious $a$ priori how the groups should be matched, but picture namingwhich is not a taxing speech task but does require both semantic and phonological processing as well as communication from the former to the latter-seemed one appropriate variable. Accordingly, the 10 PNFA cases were selected in order to achieve the closest possible pair-wise matching of cases from the two patient groups in terms of their scores on a picture-naming test from the Hodges and Patterson semantic battery (Bozeat, Lambon Ralph, Patterson, Garrard, \& Hodges, 2000), which consists of 64 line drawings of common objects from the Snodgrass and Vanderwort corpus (1980). The suitability of this variable as a basis for matching is supported by the fact that the two groups turned out to be similar on a number of other language measures, which fortunately included speech rate on a narrative task (see below). 
Many of the tests included here are either tasks for which normal accuracy would clearly be at ceiling (e.g., single-word reading or naming of common objects) or tests for which we had previously collected control data from normal participants in the age range of the two patient groups; therefore, no dedicated control group was tested for this study. Control scores from these other groups of normal participants will be reported where appropriate.

Table 1 provides demographic and background data for the two groups of patients who are ordered according to their pair-wise matching on picture naming (i.e., the first case listed under NFA was matched with the first case listed under PNFA, and so on). Not surprisingly for a contrast of CVA with progressive disease,

TABLE 1

Basic information on the patient participants

\begin{tabular}{|c|c|c|c|c|c|c|c|c|c|c|}
\hline Case & Age & Sex & $\begin{array}{l}\text { Years } \\
\text { post }\end{array}$ & Naming & $W P M$ & $P P T$ & TROG & Digits $F$ & Digits $B$ & $W C S T$ \\
\hline \multicolumn{11}{|l|}{$N F A$} \\
\hline DC & 39 & F & 2 & 64 & 64 & 52 & 71 & 6 & 1 & 6 \\
\hline JL & 48 & F & 7 & 54 & 64 & 51 & 79 & 5 & 3 & 6 \\
\hline PG & 68 & M & 3 & 53 & 63 & 51 & 71 & 4 & 0 & 5 \\
\hline JS & 47 & $\mathrm{~F}$ & 6 & 51 & 62 & 42 & 58 & 4 & 2 & 6 \\
\hline DM & 62 & M & 5 & 50 & 61 & 50 & 64 & 3 & 2 & 5 \\
\hline GN & 74 & M & 4 & 48 & 60 & 45 & 62 & 4 & 2 & 1 \\
\hline $\mathrm{AB}$ & 62 & $\mathrm{~F}$ & 7 & 42 & 62 & 47 & 55 & 3 & 2 & 3 \\
\hline MB & 85 & $\mathrm{~F}$ & 5 & 35 & 60 & 47 & 55 & 3 & 0 & 4 \\
\hline IB & 55 & $\mathrm{~F}$ & 13 & 33 & 62 & 49 & 63 & 2 & 0 & NT \\
\hline GD & 66 & $\mathrm{M}$ & 3 & 27 & 63 & 48 & 69 & 8 & 2 & 2 \\
\hline Mean & 60.6 & & & 45.3 & 62.1 & 48.2 & 64.7 & 4.2 & 1.4 & 4.2 \\
\hline$S D$ & 13.8 & & & 11.3 & 1.4 & 3.1 & 7.8 & 1.8 & 1.1 & 1.9 \\
\hline \multicolumn{11}{|l|}{$P N F A$} \\
\hline MC & 61 & $\mathrm{~F}$ & $2+1$ & 62 & 63 & 49 & 66 & 6 & 3 & 1 \\
\hline SA & 78 & M & $3+1$ & 59 & 63 & 51 & 74 & 5 & 3 & 5 \\
\hline DC & 73 & M & $4+3$ & 56 & 62 & 47 & 51 & 5 & 2 & 0 \\
\hline $\mathrm{JM}$ & 67 & M & $3+2$ & 53 & 63 & 52 & 75 & 4 & 3 & 6 \\
\hline HK & 73 & $\mathrm{~F}$ & $7+1$ & 52 & 63 & 50 & 61 & 4 & 3 & 1 \\
\hline MR & 67 & M & $2+1$ & 46 & 63 & 45 & 42 & 2 & 2 & 1 \\
\hline WK & 69 & M & $5+4$ & 44 & 63 & 49 & 64 & 3 & 2 & 0 \\
\hline DJM & 71 & M & $6+2$ & 41 & 63 & 45 & 60 & 3 & - & 2 \\
\hline $\mathrm{CH}$ & 81 & M & $4+2$ & 41 & 56 & 40 & 69 & 6 & 4 & 1 \\
\hline BR & 62 & $\mathrm{M}$ & $5+2$ & 27 & 60 & NT & 44 & 3 & 2 & 1 \\
\hline Mean & 70.2 & & & 48.1 & 61.9 & 47.6 & 60.6 & 4.1 & 2.7 & 1.8 \\
\hline$S D$ & 6.4 & & & 10.4 & 2.3 & 3.7 & 11.6 & 1.4 & 0.7 & 2 \\
\hline
\end{tabular}

"Years post" = number of years between CVA and testing for the NFA cases; for the PNFA patients, the two numbers represent the time since presentation to a neurological clinic + years of self-/carer report of symptoms prior to presentation. Naming $=$ scores out of 64 on the picture-naming test from the Cambridge Semantic Battery (controls are essentially at ceiling on this naming test); WPM $=$ scores on a 10-alternative forced-choice word-to-picture matching test on the same 64 items as in naming (controls are at ceiling on this WPM test); PPT = scores on the picture version of the Pyramids and Palm Trees 52-item test of associative knowledge (normal controls make a maximum of 3 errors on this test); TROG $=$ scores out of 80 on the Test for the Reception of Grammar (controls are near ceiling on this sentence-picture matching test); Digits $\mathrm{F}$ and $\mathrm{B}=$ each patient's digit span forwards and backwards; WCST $=$ the number of categories achieved out of 6 on the Wisconsin Card Sorting test (normal controls typically achieve all six categories). 
the NFA group had a wider age range than the PNFA patients, and on average the progressive cases were older, with a trend towards significance $(Z=1.7, p=.09)$. As one would expect given that the patient pairs were selected according to this measure, the groups did not differ on naming $(Z=0.45, p=.65)$. A simple test of comprehension for the same 64 items as those used in naming-word-picture matching in which the patients were asked to point to one of 10 semantically related pictures in response to a single spoken word - also did not distinguish between the two groups $(Z=0.36, p=.72)$, and no patient other than PNFA-case $\mathrm{CH}$ had a notable impairment on this test. A second, entirely non-verbal assessment of concept knowledge, the PPT (Pyramids and Palm Trees test: Howard \& Patterson, 1992) revealed a similar picture: no, or only mild, abnormality (with the exceptions of NFA-case JS and PNFA-case $\mathrm{CH})$, and no significant group difference $(Z=0.33$, $p=.74)$.

On a standard test of syntactic comprehension, the TROG (Test for the Reception of Grammar: Bishop, 1989), both patient groups had substantially abnormal scores (controls $=78.8, S D=1.85$ ); the PNFA cases as a group were numerically but not significantly worse than the NFA patients $(Z=0.61, p=.55)$. The two groups did not differ on forwards digit span $(Z=0.08, p=.94)$ but there was a reliable advantage in backwards digit span for PNFA relative to NFA $(Z=2.53, p=.01)$. Finally, in the non-language domain, although some of the NFA cases achieved fewer than six categories on the Wisconsin Card Sorting Test (Nelson, 1976), almost all of the NFPA patients were impaired at this standard test of frontal function, resulting in a significant group difference $(Z=2.38, p=.01)$.

\section{Experimental tasks and procedures}

Cookie Theft. This sub-test of the Boston Diagnostic Aphasia Examination (Goodglass, Kaplan, \& Barresi, 2001) was administered in the standard manner, with the participants being asked to "say everything that you can about what is going on in the picture". Descriptions were transcribed on-line but also audiotaped for subsequent checking and scoring. Descriptions were timed, beginning when the participants started to speak and terminating when they indicated that they were finished.

Exceptional Passage. This task, designed by our research group, is a 130 -word paragraph consisting of a short story about a birthday dinner. Participants were given a printed version of the paragraph and asked to read it aloud; their reading accuracy was coded by the experimenter on-line, and the whole event was timed and audiotaped for later checking. Distributed throughout the paragraph are 18 words with exceptional spelling-sound correspondences (e.g. pint, steak, suede, gloves, gauge) and, on another occasion, these individual 18 words were presented on cards to be read aloud.

\section{Reading lists.}

(1) Content vs function words. There are two solutions, neither very adequate, to the problem of comparing performance on word sets like content and function words that have minimally overlapping frequency distributions in natural language. The first is to insist on genuine frequency matching, which can only be 
done by choosing longer and less common function words such as neither and except. The second is to choose the highest-frequency content words available in a ranked frequency list like Kucera and Francis (1967), e.g., say, old, life, place, and not to fret too much about the fact that the function words are still, on average, higher in frequency. The latter was the solution adopted by Patterson (1982), and the words from that study were employed here. There are 120 items, 60 content and 60 function words matched pairwise for length; the words were printed on cards and presented in random order for oral reading.

(2) Effects of imageability. This list, consisting of 240 words, was created by Howard and Franklin (1988) to enable assessment of the impact of several different variables on reading. For current purposes, the relevant factor is that the words can be divided into subsets with high- vs low-imageability ratings (means of 5.85 vs 3.39 on a 7-point scale; examples of high = choir, glove, steam; low $=$ soul, trust, cite) with the two subsets matched for frequency (Kucera \& Francis, 1967). Again, words were printed on cards and presented in random order for oral reading.

(3) Finally, the patients were asked to read a set of 40 nonwords (from Patterson et al., 1994). They are all single-syllable strings, four or five letters in length, with common spelling patterns (e.g., kead, larp, fove). Any legitimate pronunciation-i.e., one that applies to a real word with the same spelling pattern - is accepted as a correct response; thus either $/ \mathrm{kid} /($ as in bead) or $/ \mathrm{k} \varepsilon \mathrm{d} /($ as in head) would be scored as correct for kead.

Phonological judgements/manipulations. The patients were asked to perform two tests (from Patterson \& Marcel, 1992) of phonological manipulation at the phoneme level. In the segmentation task, the participant is offered a single-syllable spoken word or nonword and asked to strip off the initial sound and say what remains. For example, the stimulus "task" should yield the response "ask", and "nisk" should yield "isk". In a second task, blending, the experimenter presents a spoken stimulus phoneme (plus schwa) that constitutes the onset of a single-syllable word or nonword and then the vowel + coda of the target response, and the participant is asked to blend these together into a single utterance. Using the same two items as examples, " $t$ " followed by "ask" should yield the response "task", and the correct response to " $n$ " + "isk" is "nisk". There were 48 trials for each of segmentation and blending; the two tasks were completed in separate sessions, and each was preceded by a substantial set of practice items because these are rather unnatural tasks. There were also two tests based on rhyme (derived from materials used by Patterson, Vargha-Khadem, \& Polkey, 1989). For the rhyme judgement task, the patient hears a sequence of 48 pairs of single-syllable spoken words, half of which rhyme (e.g., "speak-bleak") and half of which do not (e.g., "speak-break") and is asked to respond yes to rhyming pairs and no to the rest. In a rhyme production task, the patient is offered a single-syllable spoken word $(N=24)$ and asked to produce a rhyming word.

\section{RESULTS}

\section{Sensitivity of language performance to task}

We begin with some between- and within-group comparisons that are germane to the first hypothesis, namely that the deficits in PNFA are more susceptible than those in 
TABLE 2

Connected speech and success on word reading

\begin{tabular}{lccc}
\hline & NFA & PNFA & Controls \\
\hline Cookie Theft: words/min & $25.2(11.0)$ & $27.8(18.7)$ & $137.4(35.4)$ \\
Paragraph reading: words/min & $22.5(17.7)$ & $50.1(37.0)$ & $171.4(35.0)$ \\
Paragraph reading: total time (sec) & $342.0(175.1)$ & $182.5(111.2)$ & $47.0(9.8)$ \\
Exception words correct: in context & $13.0(3.4)$ & $12.4(5.7)$ & $18.0(0.0)$ \\
Exception words correct: single & $13.1(6.3)$ & $16.1(1.7)$ & $17.7(0.5)$ \\
\hline
\end{tabular}

Five measures concerning rate of connected speech (in either picture description or paragraph reading) and success on word reading in or out of context. Values in the table are means, with standard deviations in brackets. The calculation of words/min for paragraph reading is based on the number of words that each patient produced, not the number presented.

NFA to modification by the nature of the language task. Table 2 displays the following five measures: (i) speech rate (words per minute) in producing a description of the Cookie Theft picture; (ii) speech rate (ditto) in reading aloud the "exceptional passage" described above (note that the measure here is obtained for each patient by dividing the number of words that he or she produced by the total time taken to produce them; where patients omitted words, the numerator is less than 130); (iii) total time to read aloud this passage; (iv) accuracy in reading aloud the 18 words with atypical spelling-sound correspondences embedded in the exceptional passage when patients were asked to read the whole paragraph; (v) accuracy in reading aloud the same 18 words when these were presented as single words.

As one might expect from patients all classified as non-fluent, average speech rate on the Cookie Theft description (see Table 2) was markedly slow in both groups relative to normal speakers, and it did not differ between the patient groups $(Z=0.38, p=.71)$. Now consider what happened when the patients were still required to produce connected speech but did not have to generate its form or content because they were reading aloud a paragraph. The speech rate of the NFA cases in text reading was very similar to its value for self-generated speech, but the average speech rate for PNFA nearly doubled. The group difference (PNFA $>$ NFA) in words per minute for reading the paragraph reaches only a trend towards significance $(Z=1.72, p=.09)$; but total time taken was significantly less for PNFA than NFA $(Z=2.29, p=.02)$. Having the message externally specified thus facilitated speech rate in PNFA but not in NFA. Given that normal speakers read the paragraph aloud in about 40-50 seconds, the PNFA patients were certainly not completing this task in a normal amount of time; but their speech rate was task sensitive in a way that the NFA patients' speed was not.

The next relevant result concerns success in reading aloud the 18 words with atypical spelling-sound correspondences, with a shift from comparing performance in the same conditions across the two patient groups to comparing performance within groups across two conditions. The paragraph dubbed "the exceptional passage" was actually designed to determine whether patients who are impaired at reading aloud words with atypical spelling-sound correspondences when tested in standard single-word reading paradigms would produce more correct responses to such words in context than in isolation. For any type of patient or normal reader, we envisaged only two possible outcomes from this contrast for exception-word reading: 
either context $=$ single or context $>$ single. As Table 2 shows, performance of the NFA patients matched the former of these two patterns, context $=$ single $(Z=1.27$, $p=.21)$. We had not considered that performance might ever reveal a pattern of single $>$ context; but that is what the PNFA cases showed $(Z=2.53, p=.01)$. It is important to emphasise that, although this effect can only be demonstrated for the 18 exception words because they are the only items assessed both singly and in context, the same discrepancy would almost certainly apply to other words in the paragraph. That is, the implication to be drawn is not about words with an atypical spelling-sound relationship. Our conclusion from this contrast is that the requirement to produce (in this case, to read aloud) connected speech rather than single words is a sufficient struggle for patients with PNFA that it actually harms their word production.

The third pertinent contrast in this section concerns the patients' performance in another aspect of reading the whole paragraph: errors to function words. The paragraph contains 59 tokens and 20 types of function words, all extremely common words like the, and, she, with, in etc. These words are notoriously difficult for NFA patients to read (as well as to produce in spontaneous speech): this point was strikingly established by Gardner and Zurif's (1975) demonstration that such patients often succeed in reading the nouns inn and bee but fail on the function words in and be, despite the orthographic resemblance and phonological equivalence of the two pairs, and the far higher word frequency of the function words than the nouns. Accordingly, it is no surprise that the NFA patients in this study made a number of errors on the function words when reading this paragraph (see Table 3). Slightly more than half of these $(60 \%)$ were omissions and the remainder were substitutions, mostly by another function word (e.g., $a \rightarrow$ "the", was $\rightarrow$ "is", with $\rightarrow$ "in", etc). The PNFA cases also made function word errors in reading the paragraph, although their mean error rate was almost precisely half that of the NFA group. Of the total of 111 function word errors by the PNFA group in the paragraph, 74\% were omissions, and the remaining $26 \%$ were substitutions, again typically with a different function word. The striking contrast between patient groups comes not from these figures in the top row of Table 3 but rather from the comparison of row 1 with row 2 . The exact set of function words embedded in the exceptional paragraph was not given to the patients to read singly. As indicated in the Method section, however, on another occasion the patients were asked to read a set of 60 function words as single items, including most of those from the exceptional passage. As Table 3 reveals, the likelihood of function word misreadings in the NFA group was similar, indeed slightly higher, when such words were presented in isolation; but in the PNFA group, function word errors more than halved from context to single. A three-factor $\chi^{2}$ from a loglinear model demonstrated that the interaction between group and task was highly significant, $\chi^{2}(1)=39.44, p<.001$. This constitutes another indication of

TABLE 3

Error rates $(\%)$ for reading of function words in or out of context

\begin{tabular}{lll}
\hline & $N F A$ & $P N F A$ \\
\hline Error rate for function words $(N=59)$ in paragraph reading & $19 \%$ & $36 \%$ \\
Error rate for function words $(N=60)$ in list of single words & $44 \%$ & $08 \%$ \\
\hline
\end{tabular}


the enhanced difficulty of producing connected speech relative to single words for PNFA but not NFA.

\section{Nature of the phonological deficit}

The results remaining to be presented from this study all concern, from a rather broad perspective, the phonological abilities of the NFA and PNFA cases. First are accuracy measures for reading single words or nonwords aloud, displayed in Figure 1. No control data are included because normal readers are at ceiling on these tests. NFA patients almost invariably have an acquired reading disorder in the phonological-/deep-dyslexic spectrum (Balasubramanian, 1996; Caramazza, Berndt, \& Hart, 1981; Crisp \& Lambon Ralph, 2006; Friedman, 1996). The hallmark of this kind of reading impairment might be summarised in the following fashion: success in reading aloud is positively correlated with the degree of richness of semantic content in the target words to be read (Shallice \& Warrington, 1975). Thus, meaningless nonwords of even the simplest structure pose extreme difficulties; function words, whose role is more syntactic than semantic, are easier than nonwords but not much; low-imageability content words are somewhat easier than function words; and highly imageable or concrete content words yield the best performance. This rank ordering was not developed on the basis of any findings from the current study but rather from an extensive published literature on phonological and deep dyslexia (Coltheart, 1996; Coltheart, Patterson, \& Marshall, 1980). Nevertheless, as can be seen in Figure 1, the reading performance of the NFA cases in this study precisely matches the predicted rank ordering. Recall that the 60 high-frequency content words were selected to be as high as possible in frequency, as approximate mates for the 60 evenhigher-frequency function words. This criterion selects words that are rather general and thus somewhat abstract (e.g., part, fact, think).

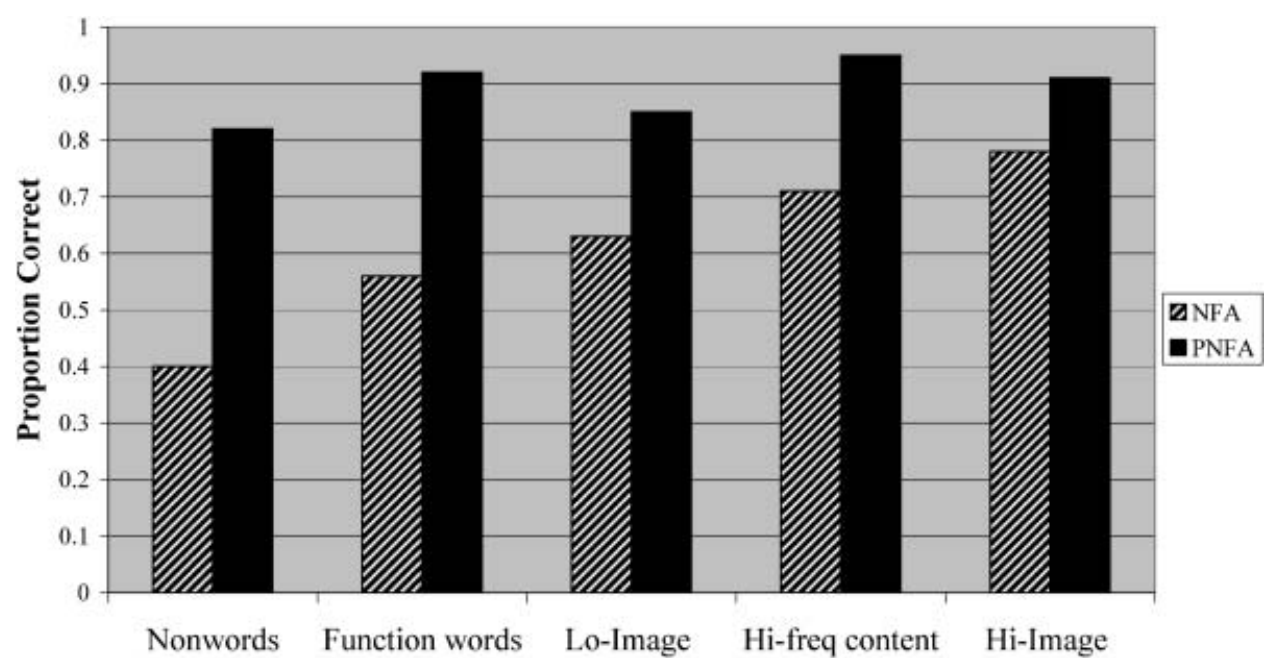

Word Type

Figure 1. Mean proportion correct for the NFA patients (slanted bars) and PNFA patients (solid black bars) on five different word-reading lists. 
If the critical variable that determines reading success in NFA is indeed semantic richness, why do we nest these results under the heading of phonological impairment? The reason is that phonological and deep dyslexia are frequently interpreted as arising from a general phonological disorder (Crisp \& Lambon Ralph, 2006; Farah, Stowe, \& Levinson, 1996; Patterson, Suzuki, \& Wydell, 1996). In the "triangle model" (Plaut, McClelland, Seidenberg, \& Patterson, 1996; Seidenberg \& McClelland, 1989), reading aloud is primarily achieved by direct activation from orthographic to phonological processing units, but semantic information about the word also contributes to activation of the spoken response. The consequence of this shared responsibility is that, when the phonological system is impaired, achieving sufficient phonological activation for response production requires a larger contribution from word meaning. Nonword reading, to which there can be essentially no contribution from word meaning, might be seen as a kind of gold (or dross!) standard for gauging impaired phonological processing.

From this perspective, a comparison of the NFA and PNFA scores on the five reading lists in Figure 1 suggests a very different quality of phonological processing in the two groups. The PNFA cases achieved significantly higher scores on four of the five stimulus sets ( $Z$ values from 2.05 to $2.98, p$ values from .04 to .003 ), the one exception being a non-significant advantage for PNFA in reading the words at which the NFA cases do best: high-imageability words. The more important point, however, is the degree to which reading varies within group across the five tests: substantially for NFA and minimally for PNFA. Note also that when difference scores between the two matched pairs of lists in Figure 1 are calculated, which is the equivalent of measuring an interaction, there was a significant group difference for high- minus low-imageability words (difference score for NFA $=15 \%$, for PNFA $=6 \%, Z=2.00, p=.04)$ and a nearly reliable difference for content minus function words (difference score for $\mathrm{NFA}=15 \%$, for $\mathrm{PNFA}=3 \%, Z=1.87$, $p=.06)$.

We now return, briefly, to performance on the exceptional passage task. As already established in Table 2, the NFA patients made an equivalent number of errors on the 18 target exception words in the two conditions, whereas for the PNFA group, the total number of errors on these target words increased from single-word to text-reading conditions. What types of errors did patients in the two groups make? Table 4 provides an error analysis for each individual patient and for each group as a whole. For single-word reading in NFA, where the majority of errors were produced by patients $\mathrm{GN}, \mathrm{AB}$, and $\mathrm{MB}$, the most common error type by far was phonological (or phonetic or articulatory: volumes have been written on how to distinguish the exact level of the speech production process responsible for errors resembling their target words in sound; we acknowledge the importance of these distinctions but our study was not addressed to this question). Many of these errors seem best described as phonological simplifications of the target (e.g., pint $\rightarrow$ /pait/; STEAK $\rightarrow /$ seik/). Apart from this main error type (or family of types) in this short test of single-word reading, the NFA patients made a few omissions, a few morphological or semantic errors (examples of the latter two types are poured $\rightarrow$ "pour" and favourite $\rightarrow$ "wonderful") and one regularisation error, where the target was pronounced as if it had a typical spelling-sound relation (mauve pronounced with the vowel as in "caught" rather than its correct vowel as in "rove"). The NFA patients" errors when reading the same words embedded in text followed a similar pattern except that a few patients (particularly $\mathrm{AB}$ ) omitted some of the target words in text reading. 
For these 18 items as single words, the PNFA patients did not make many errors but, in contrast to NFA, these were as likely to be regularisation errors (e.g., pint $\rightarrow$ /pInt/ rhyming with mint; $p u t \rightarrow / p^{\wedge} t /$ rhyming with cut) as phonological misreadings. Regularisation errors indicate good processing of the word's orthography and phonology (and the canonical relationship between them), because the word has been pronounced according to the phonological segments typically associated with that spelling pattern. Regularisations reflect not impaired phonology but failure to take account of word-specific lexical/semantic information in computing the word's correct pronunciation. The fact that regularisations, of which there was only one for the whole NFA group, were the most common error type for the PNFA group is another strand of evidence suggesting considerably different and better phonological processing in the PNFA than the NFA cases. In text reading, most errors for the PNFA patients (especially HK and DJM) were omissions, although there were still some regularisations.

Finally we come to the explicit phonological manipulation tasks of phonemic segmentation and blending, and rhyme judgement and production. The first two are somewhat unnatural tasks even for a normal speaker, and patients with phonological deficits are often completely unable to score on these tests, even when given many examples and extended practice. Indeed, perhaps the most important thing to note about the present results is this: for segmentation, 5 of the 10 NFA patients were completely unable even to attempt the task, and for blending the same 5 plus another patient (thus $N=6$ ) could not even make a start. The means given in Table 5 for NFA performance on these two tasks are therefore based on a very small number of cases (five and four respectively), and also no doubt represent the least impaired patients who were able to grasp what manipulation was needed and sometimes to perform it correctly. In the PNFA group, two patients could not attempt segmentation and one could not grasp the blending task. For what they are worth, then, the scores on these tasks are given in Table 5. Not surprisingly, with so few observations from the NFA group in particular, neither the numerical PNFA $>$ NFA advantage for segmentation nor the numerical NFA $>$ PNFA advantage for blending was statistically reliable (both $Z$ values $<1$ ). By contrast, no patient tested in either group failed to score on the two rhyming tasks; and here the advantage for PNFA $>$ NFA approached significance for rhyme judgement $(Z=1.8, p=.07)$ and achieved it for rhyme production $(Z=2.21, p=.03)$, again suggesting generally and genuinely better phonological processing in PNFA.

We argued above that the NFA patients' exaggerated sensitivity to semantic characteristics of words in reading (characteristics such as imageability or content vs function words) is a consequence of their phonological difficulties rather than reflecting a semantic impairment. This claim leads to the prediction that reading success for NFA cases should correlate much more highly with a phonological than a semantic performance measure. Now that results for the phonological measures have been presented, we can indicate that this prediction is correct. Choosing performance on two of the reading lists in Figure 1-the function words and the high-frequency content words - and correlating the NFA patients' scores on these with rhyme production as a phonological measure yields Pearson correlations of $0.92(p<.001)$ for function words and $0.86(p<.003)$ for content words. On the other hand, the relationship between reading performance on the same two lists with Pyramids and Palm Trees scores as a semantic measure is characterised by non-significant Pearson correlations: $0.32(p=.40)$ for function words and $0.25(p=.53)$ for content words. 


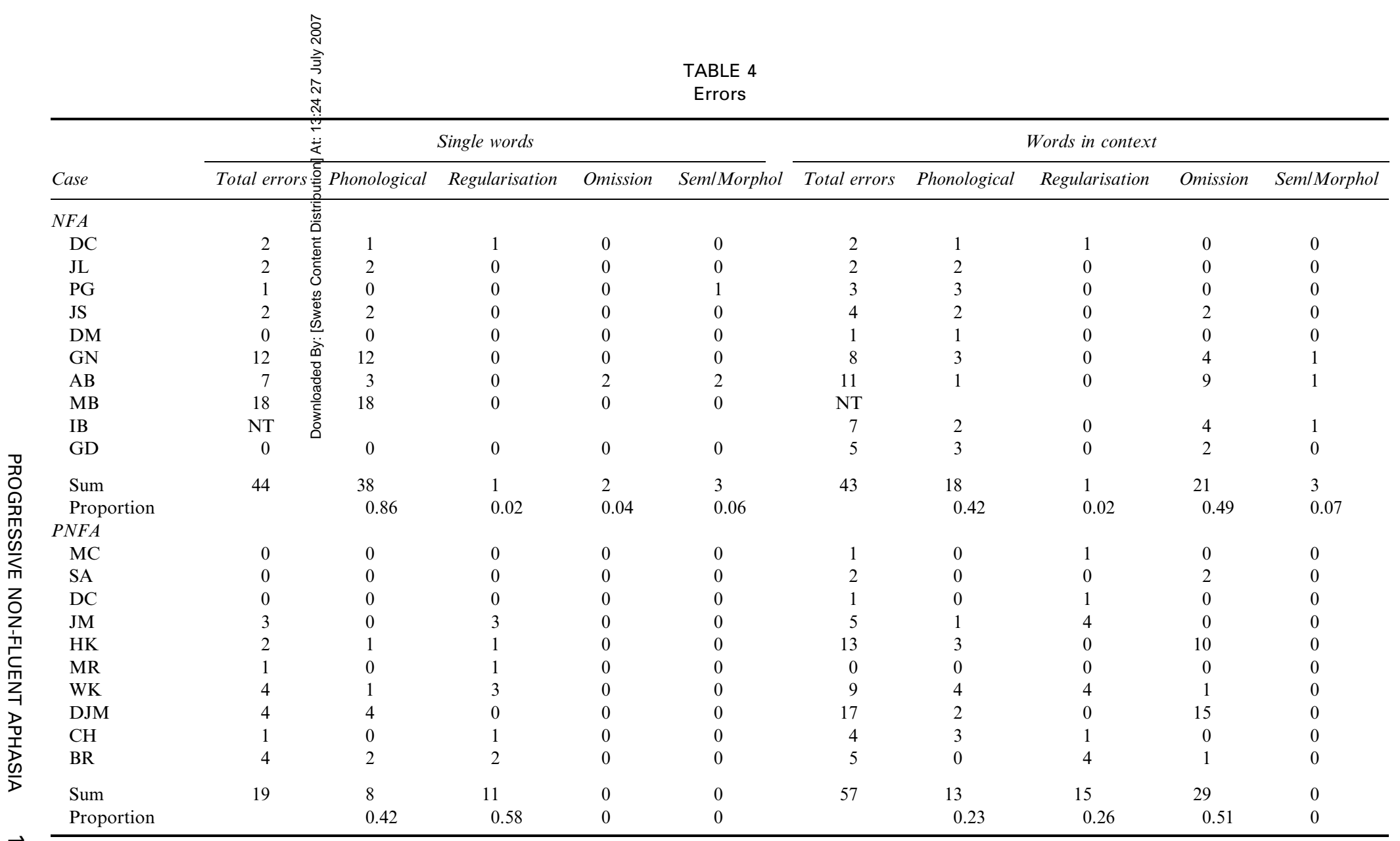

A classification of the errors in reading the set of 18 words with atypical spelling-sound correspondences presented either as single words or in the context of a short story The table provides numbers of the different errors types for each of the 20 patients plus proportions of error types for each of the two patient groups. 


\section{DISCUSSION}

The purpose of this study was to question the largely unchallenged assumption that progressive non-fluent aphasia (PNFA) arising from neurodegenerative disease is a gradually emerging facsimile of the non-fluent aphasia (NFA) often consequent on left middle cerebral artery stroke. The assumption is not a surprising one. Speech fluency is one of the main dimensions of variation in the language disorders in both abrupt-onset and slowly developing aphasia. Realistically speaking, fluency is more of a continuous than a bimodal distribution in both aetiologies, but at the two ends of the distribution, fluent and nonfluent aphasia are strikingly different. Because this is true whether the disturbance arises abruptly or gradually, it is perhaps natural to think that the similarity in spectrum equates to similarity in pattern. Furthermore, as Dame Iris Murdoch sagely commented in her novel The Black Prince, "we tame the world by generalising". There are enough similarities between NFA and PNFA that, with more than a century of information on NFA to hand, it is tempting to tame the newer world of PNFA by generalising to it what we already know about NFA. Our conclusion from this study and its sister article (Graham et al., 2004), however, is that a deeper understanding of PNFA requires attention to its differences from, as well as its similarities to, NFA.

We should note that we are aware of two considerations that might plague a prediction of theoretically relevant differences between the two patient groups. The first and more general is the inevitable heterogeneity to be found between patients, even within any single group. The second and more specific is the fact that cognitive disorders from cerebral disease or accident are not static conditions. This is of course especially true for disorders associated with neurodegenerative disease, with its inevitable decline, but it also applies to some extent to deficits following stroke, which may improve or change their character as a result of treatment, spontaneous recovery, or reorganisation of function. It is therefore possible that performance patterns in the same two patients, one from each group, could be quite discrepant at one point in time but more closely resemble each other at another time of testing. These are not completely surmountable problems, but we have at least been concerned about them and have circumvented them as best we could (a) by selecting NFA patients who were at least 2 years post-insult and hence probably past the period of most striking change, (b) by selecting PNFA patients who were neither too mild nor too severe, and (c) by matching the two groups on a pair-wise basis for picture-naming scores.

As mentioned in the Introduction, the study by Graham et al. (2004) demonstrated that the narrative speech production of a group of 14 PNFA cases

TABLE 5

Proportions correct

\begin{tabular}{lll}
\hline & NFA & PNFA \\
\hline Phoneme segmentation (48) & $0.50(0.24)$ & $0.68(0.21)$ \\
Phoneme blending (48) & $0.61(0.33)$ & $0.48(0.28)$ \\
Rhyme judgement (48) & $0.70(0.20)$ & $0.86(0.16)$ \\
Rhyme production (24) & $0.43(0.32)$ & $0.78(0.25)$ \\
\hline
\end{tabular}

Proportions correct (means, with $S D$ in brackets) for the two patient groups on four tests of phonological ability. 
was reduced in quantity, speed, and information content relative to the control participants, but was not abnormal in ratios of either content-to-function words or nouns-to-verbs. Saffran et al. (1980) provided clear examples of the severely agrammatic speech that can be observed in NFA patients, for example: "Ah ... Monday ... ah Dad and Paul ... hospital. Two ... ah doctors ... and ah ... thirty minutes ... And, er, Thursday ... ten o'clock ... doctors ... and ah ... teeth, yeah, fine" (p. 227). Such patients even tend to be agrammatic in sentence repetition, for example: "Experimenter: 'Repeat after me, "No, I do not like fish"" $\rightarrow$ Patient: 'No ... fish"” (p. 226). Graham et al. (2004) concluded that, perhaps with the exception of late-stage PNFA when patients are often approaching muteness, this frank agrammatism is not typical of PNFA.

The current study extends the differences between NFA and PNFA to two other features. First, the speech-production deficits of the group of $N=10$ NFA cases studied here were relatively unmodulated by the nature of the task, whereas those in the PNFA group were markedly more circumstance dependent. Both groups had slow and laboured speech in a self-generated narrative speech task, but when the selfgeneration requirement was withdrawn by asking the patients to read a paragraph aloud, speech rate remained the same in NFA but almost doubled in PNFA. Although the error rate was lower for PNFA than NFA, both groups made errors of omission and substitution on function words in the paragraph-reading task, but when the connected-speech component of function-word reading was withdrawn by asking the patients to read function words as single items, error rate remained the same (or even increased) in NFA but more than halved in PNFA. The two groups made equivalent numbers of errors in reading aloud a set of words with atypical spelling-sound correspondences embedded in the paragraph-reading task, but when the connected-speech requirement of this task was withdrawn by asking the patients to read the exception words as single items, error rate remained the same in NFA but dropped significantly in PNFA. It is important to emphasise that the speech output of PNFA patients is still slower and more error prone than normal even under the least demanding conditions. Nevertheless, our results suggest that, by contrast with NFA, the aphasia in PNFA is more especially a disorder of self-generated connected speech.

The second feature to which our results speak is the degree and nature of the phonological deficit. Impaired phonological ability, like impaired grammatical ability, is typically included in descriptions of PNFA as well as NFA; and at a gross level, this characterisation seems appropriate. For example, like the NFA cases in our study, the PNFA cases made some frank phonological/phonetic errors in all of the speech-production tasks. At a more detailed level of description, however, the PNFA cases revealed phonological-processing capacities far better than those of the NFA patients, in tasks like rhyme production and nonword reading. Of particular note are the following two facts about performance when the only demand was to read aloud single words. First, the PNFA cases made a number of regularisation errors to exception words; this error type, which demonstrates reasonably accurate phonological processing of the stimulus word, virtually never occurs in NFA. Second, the patients were asked to read a set of word lists varying in the amount of lexical/semantic support available (with nonwords at the low end of this dimension and high-imageability content words at the other) and hence varying inversely in the need to rely solely on phonological processing of the orthographic input. This factor substantially and monotonically predicted reading 
success for NFA patients but had a minimal and non-monotonic impact on success in PNFA.

At the risk of boring our readers, we would like to emphasise once again that, when we say "phonological processing" or "phonological difficulties", we are referring to a fairly broad range of component skills relating to language sound, including the maintenance and manipulation of phonological representations that might be best captured by the concept of auditory-verbal working memory, and also speech production processes that might be better described as phonetic encoding and/or articulation rather than phonology per se. Of course it will be vital for a growing understanding of the kinds of tasks employed here to specify exactly how they "load" on the various components under this broad rubric. And it will be vital for a growing understanding of the deficits in these patient groups to specify exactly which components might actually be responsible for a claim of "better phonological processing" in PNFA vs NFA. These are goals for future research.

We are working towards, but are some way from achieving, a satisfactory functional account of PNFA, in particular an explanation for the fact that the production of connected speech is such a (relatively selective) challenge for these patients. One clue might come from performance on the Wisconsin Card Sorting Task (Nelson, 1976). Among the ten NFA cases included here, one was not available to be tested on the WCST, five had normal scores (five to six categories), four were impaired and, of these latter four, only one patient (GN) failed to get beyond the first category. By contrast, in the PNFA group of ten, only two cases had normal scores of five or six categories; six scored only one or two; and two patients never even managed to sort consistently on a single criterion and thus had scores of zero. The PNFA patients' poor performance on this task, which is designed to measure frontal executive ability, may suggest a significant contribution of poor executive function to the patients' difficulty in connected speech production. In the meantime, as we and other researchers hunt for further clues, our interim conclusion is that the interpretation of the language disorder in PNFA will need to be at least somewhat distinct from accounts of NFA: the two disorders have some resemblances but a number of notable differences.

\section{REFERENCES}

Balasubramanian, V. (1996). Deep dyslexia and dysgraphia in a Broca's aphasic. Brain and Language, 55, 115-118.

Bird, H., Lambon Ralph, M. A., Seidenberg, M. S., McClelland, J. L., \& Patterson, K. (2003). Deficits in phonology and past-tense morphology: What's the connection? Journal of Memory and Language, 48, 502-526.

Bishop, D. V. M. (1989). Test for the Reception of Grammar (2nd ed.). London: Medical Research Council.

Bozeat, S., Lambon Ralph, M. A., Patterson, K., Garrard, P., \& Hodges, J. R. (2000). Non-verbal semantic impairment in semantic dementia. Neuropsychologia, 38, 1207-1215.

Broca, P. (1861). Remarques sur la siège de la faculté du langage articulé, suivies d'une observation d'aphemie (perte de la parole). Bulletin de la Société d'Anatomie (Paris), 36, 330-357.

Brown, J. W. (1972). Aphasia, apraxia and agnosia. Springfield, IL: Charles C. Thomas.

Caplan, D. (1987). Cambridge studies in speech science and communication. Cambridge, UK: Cambridge University Press.

Caramazza, A., Berndt, R., \& Hart, J. (1981). 'Agrammatic' reading. In F. Pirozzolo \& M. Wittrock (Eds.), Neuropsychological and cognitive processes in reading. New York: Academic Press. 
Coltheart, M. (Ed.). (1996). Phonological dyslexia [Special issue]. Cognitive Neuropsychology, 13(6).

Coltheart, M., Patterson, K., \& Marshall, J. (1980). Deep dyslexia. London: Routledge \& Kegan Paul.

Crisp, J., \& Lambon Ralph, M. A. (2006). Unlocking the nature of the phonological-deep dyslexia continuum: The keys to reading aloud are in phonology and semantics. Journal of Cognitive Neuroscience, 18, 348-362.

Farah, M. J., Stowe, R. M., \& Levinson, K. L. (1996). Phonological dyslexia: Loss of a reading-specific component of the cognitive architecture? Cognitive Neuropsychology, 13, 849-868.

Friedman, R. (1996). Recovery from deep alexia to phonological alexia: Points on a continuum. Brain and Language, 52, 114-128.

Gardner, H., \& Zurif, E. (1975). BEE but not BE: Oral reading of single words in aphasia and alexia. Neuropsychologia, 13, 181-190.

Goodglass, H., Kaplan, E., \& Barresi, B. (2001). Boston Diagnostic Aphasia Examination. Baltimore, MD: Lippincott Williams \& Wilkins.

Gorno Tempini, M., Dronkers, N., Rankin, K., Ogar, J., Phengrasamy, L., \& Rosen, H. et al. (2004). Cognition and anatomy in three variants of primary progressive aphasia. Annals of Neurology, 55, $335-346$.

Graham, N., Patterson, K., \& Hodges, J. (2004). When more yields less: Speaking and writing deficits in nonfluent progressive aphasia. Neurocase, 10, 141-155.

Grossman, M., \& Ash, S. (2005). Primary progressive aphasia: A review. Neurocase, 10, 3-18.

Grossman, M., Mickanin, J., Onishi, K., Hughes, E., D’Esposito, M., \& Ding, X-S. et al. (1996). Progressive nonfluent aphasia: Language, cognitive, and PET measures contrasted with probable Alzheimer's disease. Journal of Cognitive Neuroscience, 8, 135-154.

Hodges, J. R., \& Patterson, K. (1996). Non-fluent progressive aphasia and semantic dementia: A comparative neuropsychological study. Journal of the International Neuropsychological Society, 2, 511-524.

Howard, D., \& Franklin, S. (1988). Missing the meaning?. Cambridge, MA: MIT Press.

Howard, D., \& Patterson, K. (1992). Pyramids and Palm Trees: A test of semantic access from pictures and words. Bury St Edmunds, UK: Thames Valley Test Company.

Kertesz, A., Davidson, W., McCabe, P., Takagi, K., \& Munoz, D. (2003). Primary progressive aphasia: Diagnosis, varieties, evolution. Journal of the International Neurological Society, 9, 710-719.

Kucera, H., \& Francis, W. (1967). Computational analysis of present-day American English. Providence, RI: Brown University Press.

Mendez, M. F., Clark, D. G., Shapira, J. S., \& Cummings, J. L. (2003). Speech and language in progressive nonfluent aphasia compared with early Alzheimer's disease. Neurology, 61, 1108-1113.

Mesulam, M. (1982). Slowly progressive aphasia without generalized dementia. Annals of Neurology, 11, 592-598.

Mesulam, M. M. (2001). Primary progressive aphasia. Annals of Neurology, 49(4), 425-432.

Nelson, H. E. (1976). A modified card sorting test sensitive to frontal lobe defects. Cortex, 12, 313-324.

Nestor, P. J., Graham, N. L., Fryer, T. D., Williams, G. B., Patterson, K., \& Hodges, J. (2003). Progressive non-fluent aphasia is associated with hypometabolism centred on the left anterior insula. Brain, 126, 2406-2418.

Patterson, K. (1982). The relation between reading and phonological coding: Further neuropsychological observations. In A. W. Ellis (Ed.), Normality and pathology in cognitive functions. London: Academic Press.

Patterson, K., Graham, N., \& Hodges, J. (1994). Reading in dementia of the Alzheimer type: A preserved ability? Neuropsychology, 8(3), 395-407.

Patterson, K., \& Marcel, A. (1992). Phonological ALEXIA or PHONOLOGICAL alexia? In J. Alegria, D. Holender, J. Junca de Morais, \& M. Radeau (Eds.), Analytic approaches to human cognition. Amsterdam: North Holland.

Patterson, K., Suzuki, T., \& Wydell, T. N. (1996). Interpreting a case of Japanese phonological alexia: The key is in phonology. Cognitive Neuropsychology, 13, 803-822.

Patterson, K., Vargha-Khadem, F., \& Polkey, C. E. (1989). Reading with one hemisphere. Brain, 112, 39-63.

Plaut, D. C., McClelland, J. D., Seidenberg, M. S., \& Patterson, K. (1996). Understanding normal and impaired word reading: Computational principles in quasi-regular domains. Psychological Review, 103, 56-115. 
Saffran, E., Schwartz, M., \& Marin, O. (1980). Evidence from aphasia: Isolating the components of a production model. In B. Butterworth (Ed.), Language production (Vol. 1). London: Academic Press.

Seidenberg, M. S., \& McClelland, J. L. (1989). A distributed, developmental model of word recognition and naming. Psychological Review, 96, 523-568.

Shallice, T., \& Warrington, E. (1975). Word recognition in a phonemic dyslexic patient. Quarterly Journal of Experimental Psychology, 27, 187-199.

Snodgrass, J., \& Vanderwart, M. (1980). A standardized set of 260 pictures: Norms for name agreement, image agreement, familiarity, and visual complexity. Journal of Experimental Psychology: Human Learning and Memory, 6, 174-215.

Snowden, J. S., Neary, D., \& Mann, D. (1996). Frontotemporal lobar degeneration: frontotemporal dementia, progressive aphasia, semantic dementia. New York: Churchill Livingstone.

Thompson, C. K., Ballard, K. J., Tait, M. E., Weintraub, S., \& Mesulam, M. (1997). Patterns of language decline in non-fluent primary progressive aphasia. Aphasiology, 11(4), 297-321. 